For instance, if the Ryves ring is only visible in direct and brilliant sunshine, that will be evidence in favour of the shado: theory. If it is equally visible when the sun is not actually shining, but when the sky is illuminated by numerous white clouds, that will be evidence of the grass theory.

I may add that our observations were made from the west, and at noon.

C. V. Boys.

\section{An Edinburgh Record of the Indian Earthquake.}

A VERY interesting record of the recent earthquake at Calcutta is shown by the photographic apparatus of the bifilar pendulum of this observatory. A few very slight preliminary tremors commenced June II, at $23 \mathrm{~h}$. I8m. G. M.T., and lasted for ten minutes. Violent oscillations then commenced suddenly, and lasted to June 12 , oh. $33 \mathrm{~m}$., after which slighter tremors

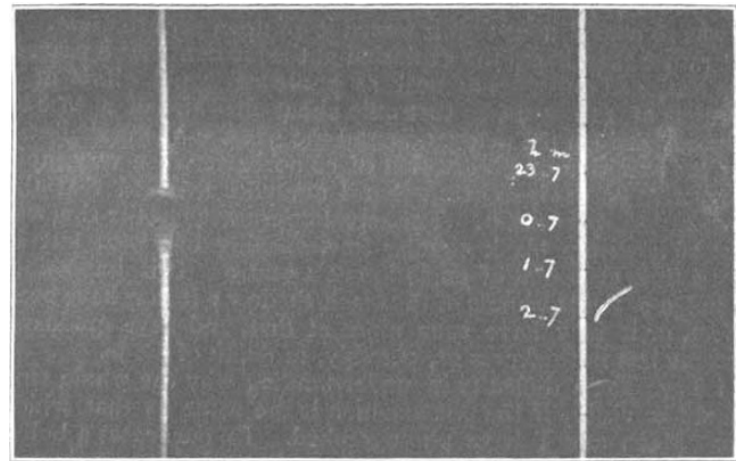

continued up to Ih. $12 \mathrm{~m}$. The oscillations can be traced fully six times the measure of sensitiveness of the instrument on one side of the normal line, and four times on the other, which are together equivalent to a tilt of the supporting frame of about twenty seconds of arc. I enclose a negative of the original photograph, which, however, does not show all the minor details of the effect produced.

Royal Observatory, Edinburgh, June 15.

\section{Subjective Transformations of Colour.}

Mr. SHELFORD BIDWELL's experiments, described in NATURE of June Io (p. 128), remind me of a phenomenon which can be very easily demonstrated.

A disc is arranged so that a small sector, about one-sixth, is of a bright colour, while the remaining portion is white. If this be rotated slowly, the coloured sector appears to be followed by a ghost of the complementary colour: on quickening the rotation, the original colour is lost, and the whole disc appears to be of the complementary colour; but if the rotation be further quickened until flickering ceases, the original colour again predominates. In this way emerald-green may appear to change to pink, or crimson-lake to green.

This seems to be another instance in which the negative aftersensation is stronger than the original sensation. For the success of the experiment it is probably necessary so to adjust the rotation that $(a)$ the negative sensation has a longer duration than the original sensation, and (b) the next original stimulus shall follow before the after-sensation has entirely faded. If the rotation be too rapid, the negative sensation has not time to develop, and only the original colour is seen.

Mason College, June I2.

$$
\text { F. J. Allen. }
$$

\section{Planetary Orbits, illustrated by a Rolling Ball.}

The interesting article in Nature, April 29, by R. W. Wood, on the orbits of a steel ball about a magnet pole, suggests to me that it may interest some of your readers to hear of another plan for showing these orbits, which, but for the slight resistance of the air, is very nearly theoretically accurate, and in which the proper initial velocities are easily produced.

The plan consists of causing a true steel ball-of, say, one inch or more in diameter-to roll on a hard and smooth surface of the proper form. The surface on which the centre of the ball moves is formed by the revolution of a part of a rectangular hyperbola about a vertical asymptote, and the real surface on which the ball rolls is, of course, a distance equal to the radius of the ball from this imaginary surface at all points.

Fig. $I$ is a vertical section, on a very small scale, in which the dotted lines show the rectangular hyperbola and its vertical and horizontal asymptotes, and the full curved lines show the actual surface on which the ball rolls; the interval between the dotted and full curved lines being equal to the radius of the ball.

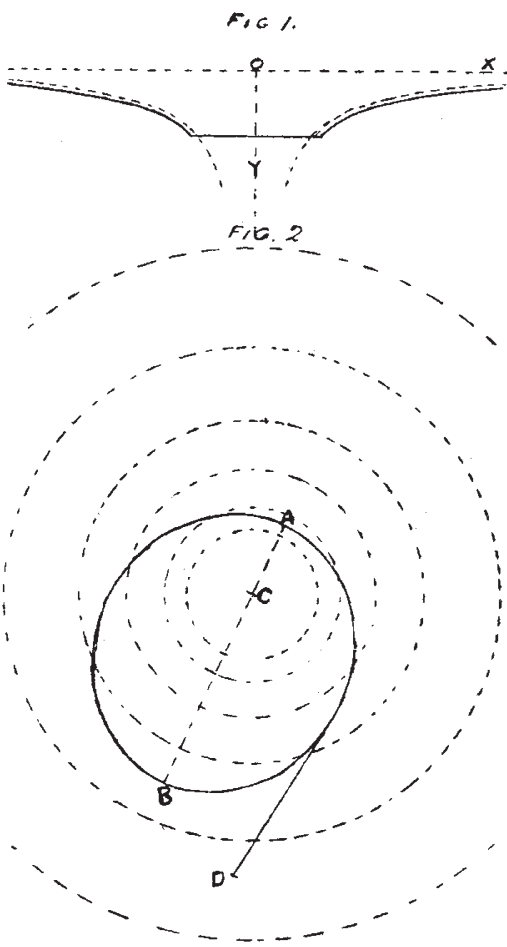

Fig. 2 is a projection on a horizontal plane showing contours of the hyperbolic surface at equal vertical intervals in dotted lines, and also one of the orbits referred to below in full lines.

Suppose the ball to be allowed to roll from the level of the horizontal asymptote, and directed so as not to come too near the centre (C) of the surface, it will describe a path whose projection on a horizontal plane is very nearly a parabola; and-as in the imaginary case of a comet coming from an infinite distance without initial velocity-its energy will be accurately in the inverse proportion to its horizontal distance from the centre of attraction, because the reciprocal of its distance from the centre is proportional to the vertical distance it has fallen, by the weilknown property of the rectangular hyperbola. If the ball be allowed to roll from a point higher than the horizontal asymptote, it will describe a hyperbola; and if fron: a lower point, an ellipse.

To produce an ellipse having any given axis-major as A B, Fig. 2, let the ball roll from a point D. on the hyperbolic surface, whose distance from the centre $C$ is equal to $A \mathrm{~B}$, and let it be directed with a straight-edge till it touches the desired orbit.

If we could neglect the resistance of the air and all other small resistances to the rolling of the ball, the actual energy, and therefore the velocity of the ball would be precisely what they ought to be to illustrate planetary motion; the direction of motion not being generally horizontal, however, and the orbit not exactly in one plane.

The fact that a part of a rolling ball's energy is rotational and part translational does not vitiate the experiment, because the proportion of the one part of the energy to the other remains constant.

In the case of a steel ball attracted by a magnet pole, the acceleration would appear to be inversely as some higher power of the distance than the square. In the case explained above, the acceleration is very nearly inversely as the square of the horizontal distance from the centre, and only differs from this proportion in being less, as the actual distance traversed by the ball exceeds its horizontal component, at any point ; which may be a very small percentage.

Craigknowe, Slateford, Midlothian. 